\title{
The skeletal muscle circadian clock: current insights
}

This article was published in the following Dove Press journal:

ChronoPhysiology and Therapy

13 November 2017

Number of times this article has been viewed

\author{
Reiko Nakao' \\ Takeshi Nikawa ${ }^{2}$ \\ Katsutaka Oishi ${ }^{1,3,4}$ \\ 'Biological Clock Research Group, \\ Biomedical Research Institute, \\ National Institute of Advanced \\ Industrial Science and Technology \\ (AIST), Tsukuba, ${ }^{2}$ Department of \\ Nutritional Physiology, Institute \\ of Biomedical Sciences, Tokushima \\ University Graduate School, \\ Tokushima, ${ }^{3}$ Department of Applied \\ Biological Science, Graduate \\ School of Science and Technology, \\ Tokyo University of Science, Noda, \\ ${ }^{4}$ Department of Computational and \\ Medical Sciences, Graduate School of \\ Frontier Sciences, the University of \\ Tokyo, Kashiwa, Japan
}

Abstract: Skeletal muscle functions in locomotion, postural support, and energy metabolism. The loss of skeletal muscle mass and function leads to diseases such as sarcopenia and metabolic disorders. Inactivity (lack of exercise) and an imbalanced diet (increased fat or decreased protein intake) are thought to be involved in the prevalence of such pathologies. On the other hand, recent epidemiological studies of humans have suggested that circadian disruption caused by shift work, jet lag, and sleep disorders is associated with obesity and metabolic syndrome. Experimental studies of mice deficient in clock genes have also identified skeletal muscle defects, suggesting a molecular link between circadian clock machinery and skeletal muscle physiology. Furthermore, accumulating evidence about chronotherapy, including chronopharmacology, chrononutrition, and chronoexercise, has indicated that timing is important to optimize medical intervention for various diseases. The present review addresses current understanding of the functional roles of the molecular clock with respect to skeletal muscle and the potential of chronotherapy for diseases associated with skeletal muscle.

Keywords: biological rhythm, metabolic syndrome, physical activity, neural signal, chronotherapy

\section{Introduction}

Skeletal muscle comprises $40 \%$ of the body mass and it functions in locomotion and postural support and as a major metabolic organ that contributes to basal metabolism, glucose uptake, and fatty acid oxidation. Balanced muscle protein synthesis and degradation can retain skeletal muscle mass and muscle performance. Muscle protein metabolism varies according to nutritional and environmental circumstances, increasing in response to exercise, food (protein) intake, and anabolic hormones such as testosterone, insulin, and insulin-like growth factor 1 and decreasing in response to aging, starvation, cancer, diabetes, immobility, loss of neural input, and catabolic hormones such as glucocorticoids. ${ }^{1}$ Compromising muscle protein metabolism results in a net loss of muscle mass and power, which prevents optimal muscle function, leading to sarcopenia, diabetes, and obesity. ${ }^{1}$ Conventional methods to stimulate muscle protein synthesis and prevent muscle protein degradation focus on the intake of adequate dietary protein and regular exercise. However, Yu et al recently found that the prevalence of diabetes, metabolic syndrome, and sarcopenia is $8.2 \%, 7.4 \%$, and $6.7 \%$ higher, respectively, in individuals classified as having an evening, rather than a morning, chronotype among middle-aged Korean population, although the average age was lower in individuals with an evening, rather than a morning, chronotype. ${ }^{2}$ Although
Correspondence: Katsutaka Oishi Biological Clock Research Group, Biomedical Research Institute, National Institute of Advanced Industrial Science and Technology, Central 6, I-I-I Higashi, Tsukuba, Ibaraki 305 8566, Japan

$\mathrm{Tel} / \mathrm{Fax}+81298616053$

Email k-ooishi@aist.go.jp 
a direct relationship between shiftwork and loss of muscle mass and strength has not been established, the disruption of circadian rhythms is found to be associated with obesity and metabolic syndrome in shift workers. ${ }^{3}$ These findings suggest that skeletal muscle homeostasis might be disturbed not only by a lack of exercise and low intake of dietary protein but also by the disruption of circadian rhythm circumstances such as continuous night work, shift work, lack of sleep, and jet lag. This review explores the potential molecular mechanisms that link the circadian clock machinery with skeletal muscle physiology and discusses the potential therapeutic implications for disturbed skeletal muscle physiology associated with pathologies such as sarcopenia and diabetes based on the circadian system.

\section{Circadian clock machinery Organization of the central clock}

Circadian rhythms are evolutionally conserved, $24 \mathrm{~h}$ biological cycles that prepare life forms ranging from bacteria to plants and mammals for daily environmental fluctuations. An internal circadian machinery controls various physiological and behavioral rhythms such as sleep/wake cycles, body temperature, metabolism, and hormone secretion. This mechanism functions to optimize the timing of cellular events in anticipation of environmental changes such as daylight, food availability, and predator/prey interactions. The mammalian master clock is located in the suprachiasmatic nucleus (SCN) of the anterior hypothalamus. ${ }^{4-6}$ The dominant environmental time cue for circadian clocks is light, which is collected via the retinohypothalamic tract and input into the SCN, where the master clock synchronizes an endogenous circadian timing system to a 24-h light-dark cycle. ${ }^{7,8}$ Molecular studies suggest that transcriptional feedback loops comprising the periodic expression of clock genes drive the circadian oscillator in the SCN. ${ }^{9}$ The positive loop of the core clock is formed by the basic helix-loop-helix-PER-ARNT-SIM (bHLH-PAS) transcription factors, CLOCK and BMAL1 ${ }^{9,10}$ CLOCK and BMAL1 form heterodimers and transactivate other clock genes such as Per1, Per2, Cry1, and Cry2 via E-box elements in their promoters. ${ }^{9,10}$ These molecules are referred to as core molecular components. The resulting PER and CRY proteins localize in the cytoplasm, where the accumulation of PER1/2 and CRY1/2 promotes the formation of PERs/CRYs/CKIE complexes ${ }^{11}$ that translocate to the nucleus and interact with CLOCK-BMAL1 complexes to inhibit Per and Cry gene transcription. ${ }^{12}$ Thereafter, CLOCK-BMAL1 can activate a new cycle of transcription. These processes are considered to comprise the main feedback loop of the molecular clock and $\sim 24 \mathrm{~h}$ are needed to complete one feedback cycle. The posttranslational modification and degradation of molecular clock proteins are also important for determining circadian periodicity. Accumulated PER1/2 proteins are phosphorylated in the cytoplasm by CK1 $1 .{ }^{13}$ Phosphorylated PER1/2 is a target for polyubiquitination via the function of $\beta$-TrCP ubiquitin ligase and degradation by the $26 \mathrm{~S}$ proteasome. ${ }^{14}$ Cytoplasmic CRY 1/2 proteins are also degraded by the ubiquitination system mediated via FBXL3 ubiquitin ligase. ${ }^{15}$ CLOCK-BMAL1 also initiates the transcription of a second feedback loop that is involved in the E-box-mediated transcription of $R O R a$ and Rev-erba. ${ }^{16,17} \mathrm{REV}$-ERB $\alpha$ protein can repress Clock and Bmall via RORE transcription sequences located within their promoters, whereas ROR $\alpha$ can activate Bmall transcription. ${ }^{16,18,19}$

\section{Peripheral clocks}

Core clock components have been identified in most peripheral organs including the lungs, liver, and skeletal muscle, indicating that circadian oscillators (called peripheral clocks) are located in these tissues. ${ }^{20-22}$ Comprehensive gene analysis of the liver using DNA microarrays has shown that molecular clock components regulate the expression of hundreds of genes outside the feedback loop. ${ }^{23}$ These genes are called clock-controlled genes and they are direct transcriptional targets of the core clock machinery. ${ }^{23,24}$ Comparisons of circadian transcriptomes among tissues have indicated a low ratio of circadian genes that are commonly expressed in various tissues..$^{23,25,26}$ This tissue specificity of circadian genes is considered to govern the physiological characteristics of each tissue.

\section{Discovery of molecular clocks in skeletal muscle}

Zylka et al discovered the circadian expression of Per $1 / 2 / 3$ in skeletal muscle and proposed that circadian oscillators reside in skeletal muscle. ${ }^{27}$ To identify transcripts with circadian expression in skeletal muscle, Miller et $\mathrm{al}^{26}$ and McCarthy et $\mathrm{a}^{28}$ independently used DNA microarrays to find 267 and 215 genes, respectively, with expression that oscillated over a $24 \mathrm{~h}$ period. Among them, some genes belonged to the core molecular clock, whereas others were specific to muscles and involved in metabolism (ATP synthases, Fbxo32 also known as Mafbx or Atrogin-1, and/or Trim63 also known as Murf1), transcription (Myod1), and cytoskeletal organization (Troponins and Myhl). ${ }^{28}$ The profiling of circadian gene expression has provided basic evidence with which to understand the role of the molecular clock in skeletal muscle. 


\section{Synchronization of circadian rhythms in skeletal muscle}

A critical feature of peripheral clocks is that the phase of circadian clock gene expression can be synchronized with the SCN, which was originally thought to have a masterslave relationship with peripheral clocks by coordinating their respective circadian rhythms via neural and humoral signals. ${ }^{29,30}$ However, more recent studies have found that scheduled feeding or exercise can phase-dissociate the molecular clock in peripheral tissues from that in the $\mathrm{SCN}^{31,32}$ The dominant stimuli for synchronization of the skeletal muscle clock to the SCN remain controversial. When SCN-lesioned and intact mice were parabiotically linked and shared a circulatory system, humoral and behavioral signals could not reinstate the circadian expression of clock genes in skeletal muscle, heart, and spleen, but they resynchronized clock gene expression in the liver and kidney, suggesting that humoral signals are insufficient to enforce rhythmicity in skeletal muscle. ${ }^{33}$ This section discusses the intensity of feeding and exercise as entrainment factors for skeletal muscle clocks.

\section{Function of feeding and humoral factors on circadian expression of clock genes in skeletal muscle}

Feeding time is a dominant zeitgeber for peripheral clocks in mammals. Exposing nocturnal rodents to time-imposed restricted feeding (RF) for $4 \mathrm{~h} /$ day during the light period inverts the circadian phase of clock gene expression in the liver but does not affect that in the SCN..$^{31,32,34}$ The possibility that insulin causes feeding-induced phase shifts in clock gene expression within peripheral tissues has been considered. We found that the temporal expression of circadian clock genes in mice became synchronized in the liver, but not in skeletal muscle when feeding is restricted to $8 \mathrm{~h} /$ day during the light period. ${ }^{35,36}$ We also found that RF inverts the circadian phase of plasma insulin, ${ }^{35,36}$ suggesting that the circadian fluctuation of plasma insulin is not a dominant zeitgeber for the skeletal muscle clock. Nocturnal wheelrunning activity, body temperature, and corticosterone secretion were similar between mice fed only during the nighttime or the daytime under our experimental conditions. ${ }^{35}$ Neural signals from the central clock in the SCN, corticosterone, physical activity, and body temperature might be more potent zeitgebers for the skeletal muscle clock than feeding time and insulin, although intraperitoneally injected insulin induces Per 1 and Per 2 gene expression in mouse skeletal muscle. ${ }^{36}$ Differences in tissue sensitivity to feeding time might cause desynchronization among molecular clocks in metabolic organs such as the liver and skeletal muscle, leading to disrupted metabolism.

\section{Impact of physical activity on circadian clock gene expression in skeletal muscle}

Exercise is also a time cue for skeletal muscle clocks that is independent of the SCN. Several studies have shown that scheduled wheel running or treadmill exercise during the inactive period advances the phase of the circadian activity rhythm in rodents. ${ }^{37,38}$ Zambon et al reported that the human clock genes, PER 2 and BMAL1, are upregulated by resistance training in one leg compared with the contralateral untrained leg. ${ }^{39}$ McCarthy et al identified 215 circadian genes in a DNA microarray analysis of mouse skeletal muscle, among which expression peaked in $40 \%$ of them at the middle of the dark (active) period. ${ }^{28}$ Wolff and Esser showed that the circadian phase of bioluminescence rhythms in the skeletal muscle of PER2-Luciferase knock-in mice was advanced $3 \mathrm{~h}$ by forced treadmill exercise for 4 weeks during the light (inactive) period, but the SCN was not affected. ${ }^{40}$ These findings indicated that muscle contractile activity might be a zeitgeber in skeletal muscle independently of the SCN. However, exercise is accompanied by systemic changes, such as accelerated energy metabolism, hormonal fluctuations, increased body or local muscle temperature, and neural adaptation, ${ }^{41}$ and which factor is critical for resetting muscle clocks has not been determined. Glucocorticoids are critical time cues for peripheral clocks ${ }^{42}$ and their release is increased by exercise. We have shown that the peak plasma corticosterone levels in mice given free access to a running wheel for 4 weeks were twice as high as those in mice housed under sedentary conditions, and temporal profiles of corticosterone concentration were phase advanced in mice housed with a running wheel. ${ }^{43,44}$ We also have shown that phase advance of corticosterone concentration coincided with the elevation of peak expression level and slight phase advance of circadian clock gene expression in skeletal muscle of mice housed with a running wheel ${ }^{43}$ Sasaki et al showed that forced treadmill running increased serum corticosterone concentration and it seemed to contribute to the phase shift of peripheral clocks. ${ }^{45}$ These findings suggest that corticosterone secretion might be a potent zeitgeber in skeletal muscle during exercise. We previously reported that neural signals are indispensable to retain the normal oscillation of clock 
gene expression in the skeletal muscle of mice with sciatic denervation. ${ }^{46}$ The expression levels of most clock genes such as Bmal1, Per1, Rev-erba, and Dbp were reduced in denervated muscle, although circadian Per2 expression was significantly augmented (Figure 1). The circadian expression of Bmall and Dbp is phase advanced in denervated muscle. ${ }^{46}$ Dyar et al also recently found that Bmall and $D b p$ are phase advanced in denervated muscle and they interpreted this as resulting from total muscle inactivity. ${ }^{47}$ Sciatic denervation did not affect clock gene expression in the contralateral muscle (Figure 1), indicating that humoral changes are not involved in muscle clock disruption induced by denervation. Sciatic denervation did not alter the circadian rhythms of body temperature, spontaneous behavior rhythms, corticosterone concentrations, and hepatic clock gene expression, indicating that systemic circadian rhythms do not affect disrupted clock gene expression in denervated muscle. ${ }^{46}$ However, whether the effect on atypical clock gene expression depends upon the loss of nerve activity or muscle contraction is not understood. We postulate that the activity of nerves and accompanying muscle contraction are more potent synchronizers for skeletal muscle than glucocorticoids during exercise (Figure 2).

\section{Functional roles of the molecular clock and clock-controlled genes in skeletal muscle Phenotype of muscle mass and strength in clock gene-mutant mice}

Kondratov et al discovered that the molecular clock is important for maintaining skeletal muscle homeostasis. ${ }^{48}$ They reported that the life span of Bmall-null mice is short and they develop severe sarcopenia at 40 weeks of age, whereas these phenomena are not evident in wild-type mice. ${ }^{48}$ Andrews et al demonstrated reduced muscle force, dysfunctional mitochondria, and disrupted myofilament architecture in Bmall-null mice and $\mathrm{Clk} / \mathrm{Clk}$ mutant mice, suggesting that disruption of the molecular clock leads to structural and functional changes in skeletal muscle. ${ }^{49}$
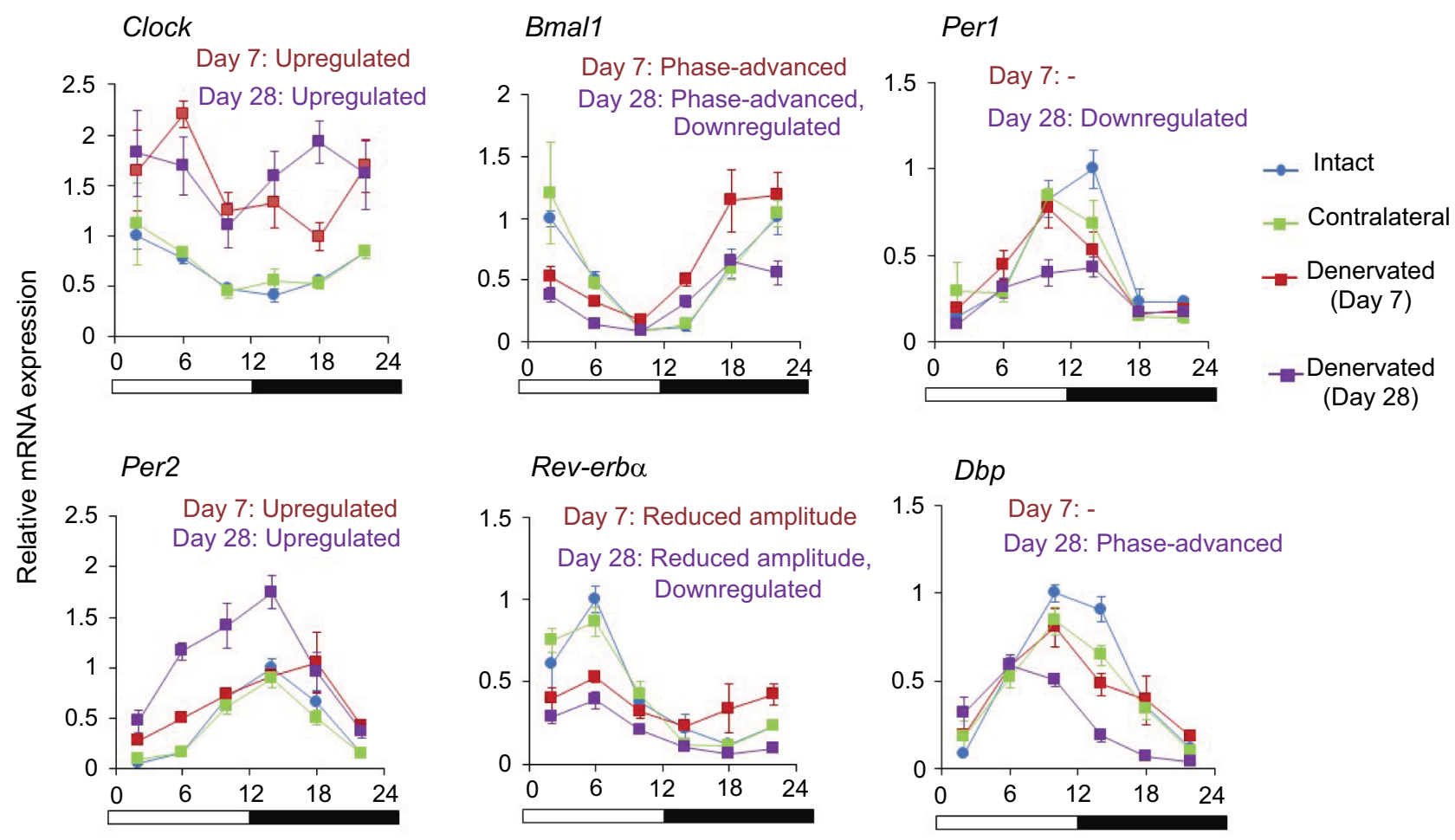

Zeitgeber time(h)

Figure I Sciatic denervation disrupts circadian rhythms of clock gene expression in skeletal muscle on days 7 and 28 after denervation.

Notes: Messenger RNA expression of clock genes in skeletal muscle at indicated times. Sciatic nerve was unilaterally transected and mice were sacrificed at $4 \mathrm{~h}$ intervals 7 and 28 days later. Contralateral innervated muscles of the same mice and muscles of intact mice served as controls. Sciatic denervation is associated with robust and sequential muscle atrophy. Denervated muscle weight decreased by $30 \%$ and $67 \%$ at days 7 and 28 , respectively, compared with intact muscle. ${ }^{46} \mathrm{Upregulated} \mathrm{Clock} \mathrm{expression}$ was not associated with progressive muscle atrophy. Expression of Per2, Bmall, and Rev-erba was atypical on days 7 and 28 and became altered as atrophy advanced. Expression of Perl and Dbp was atypical only on day 28 (when muscle had become more atrophic). White and black bars below each graph indicate light and dark periods. ' ' indicates no effect. Data from Nakao et al. ${ }^{46}$ 


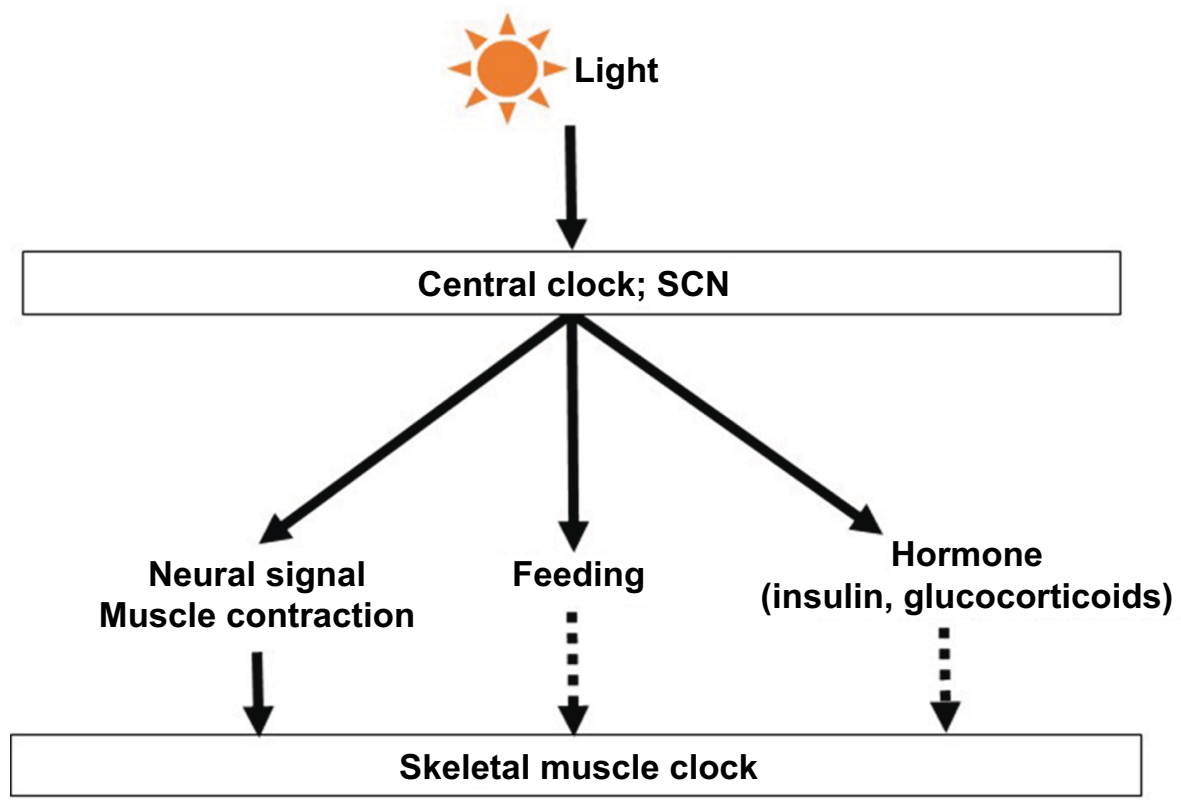

Figure 2 Several zeitgebers work for appropriate oscillation of skeletal muscle clock. Light input synchronizes master circadian clock in the SCN to environmental light-dark cycles, followed by aligned behavioral and physiological rhythms. Peripheral tissues differ in terms of dependence on time cues such as neural or humoral factors. Skeletal muscle seems more sensitive to neural signals and muscle contraction than to feeding and humoral factors.

Abbreviation: SCN, suprachiasmatic nucleus.

The phenotype of skeletal muscle is heavily influenced by systemic factors such as hormones, metabolic state, activity level, and neural input. The possibility that the altered skeletal muscle in Bmall-null mice and Clk/Clk mutant mice resulted from a clock gene mutation in other organs could not be excluded. Dyar et al examined the specific role of the skeletal muscle clock using mouse models in which Bmall in skeletal muscle was specifically abolished. ${ }^{50}$ The line was a traditional tissue-specific knockout (KO); that is, mice with a floxed Bmall allele were bred with mice carrying a Cre recombinase transgene under the control of the Mlclf promoter. $^{50}$ Unlike Bmal1-null mice, these muscle-specific Bmall KO mice did not develop sarcomere changes or degeneration even at the age of 26 months. ${ }^{50}$ In addition, the muscle of these mutants was heavier, although with slightly less force than those of wild-type mice at 5 months age. ${ }^{50} \mathrm{We}$ also reported that the muscle weight of wild-type and muscle-specific Bmall KO mice was essentially identical in both adult (7-8 months) and aged (23-24 months) mice. ${ }^{51}$ The extent of muscle mass loss induced by sciatic denervation was also similar between wildtype and muscle-specific Bmall KO mice, although Bmall ablation partly attenuated the upregulation of genes encoding the muscle atrophy-related ubiquitin ligases, MAFbx and MuRF1, indicating that muscle Bmall plays a minimal role in the regulation of muscle mass loss..$^{51}$ These results suggested that the circadian clock in organs other than skeletal muscle causes the aging phenotype in Bmall-null mice.
Dyar et al further generated an inducible muscle-specific Bmall KO (im-Bmal1 KO) line to eliminate any effects of the Bmall deletion on skeletal muscle development and postnatal maturation. These mice were generated by crossing floxed Bmall mice with HSA-Cre-ER mice, in which the human skeletal actin promoter controls a Cre recombinase fused with a mutated estrogen receptor that can be activated by tamoxifen. ${ }^{50}$ Dyar et al found no differences in muscle weight and force between the two types of mice after 5 months of tamoxifen administration..$^{50}$ On the other hand, Schroder et al found reduced specific tension and increased muscle fibrosis in a similar im-Bmall $\mathrm{KO}$ model after 58 weeks of tamoxifen treatment. ${ }^{52}$ They also found significant bone calcification and decreased joint collagen at that age, suggesting that the muscle clock functions in both skeletal muscle and systemic tissues via an endocrine/paracrine function. ${ }^{52}$ Regarding the discrepancies in the results of the studies by Dyar et al and Schroder et al, Schroder et al noted that differences in experimental design might have been the cause. ${ }^{52}$ Tamoxifen was injected into mice at 8 weeks of age (Dyar et al), when satellite cell fusion to myofibers is still enhanced, and into mice aged between 12 and 16 weeks (Schroder et al). Schroder et al estimated that in Dyar et al's study, Bmall-negative myonuclei could have been diluted by adding Bmall-positive nuclei after tamoxifen administration. ${ }^{52}$ Regardless, the Bmall mRNA content in skeletal muscle remarkably decreased in both studies. The second 
difference that Schroder et al pointed out was the age of mice when they were analyzed. Dyar et al found no difference in muscle force between im-Bmall $\mathrm{KO}$ and wild-type mice after 5 months (20 weeks) of tamoxifen treatment, while Schroder et al analyzed muscle phenotype of im-Bmall KO mice after 56 weeks of tamoxifen treatment. We estimated that the effect of Bmall deletion might be compensated by unknown molecules or other organs in younger mice. Aging leads to systemic changes such as denervation of motor units, anabolic resistance, and reduced blood flow, as well as defects in mitochondria and other organelles. ${ }^{53,54}$ Molecular clock disruption accelerates such changes. For example, hepatic Bmall gene deletion elevates oxidative damage associated with abnormal mitochondrial morphology, although information about oxidative stress in muscle-specific Bmall $\mathrm{KO}$ mice is not available. ${ }^{55}$ Aging and molecular clock disruption might additively increase health risk for skeletal muscle in aged im-Bmall $\mathrm{KO}$ mice.

Yang et al recently generated ubiquitously inducible Bmall KO mice by crossing a floxed Bmall line with a tamoxifen-inducible universal Cre line. ${ }^{56}$ Tamoxifen started at the age of 3 months inactivated Bmall in all tissues in adult mice. Life span, body weight, organ weight including muscle, fertility, blood glucose levels, and arthropathy did not significantly differ between Bmall KO and Bmal1-positive mice. These findings suggested that the extreme aging phenotype in Bmall null mice was caused by Bmall function during development. ${ }^{56}$ The functional role of Bmal1 during development remains unknown. It might play a role independently of its transcriptional rhythmicity because its expression does not oscillate throughout the day in embryonic tissues. ${ }^{57}$ Indeed, a recent study found that BMAL1 rhythmically interacts with translational machinery and promotes protein synthesis in the mouse liver and brain during the active period independently of its role as a transcription factor. ${ }^{58}$ To identify the role of Bmall in protein synthesis in the skeletal muscle of adult mammals requires further study.

\section{Metabolic phenotype of muscle-specific Bmall-deficient mice}

Studies of skeletal muscle-specific Bmall KO mice have focused on metabolic effects. Glucose uptake in the skeletal muscles of muscle-specific Bmall $\mathrm{KO}$ mice is impaired with reduced protein levels of GLUT4, an insulin-dependent glucose transporter, and TBC1D1, a Rab-GTPase involved in GLUT4 translocation. ${ }^{50}$ The activity of pyruvate dehydrogenase that catalyzes the decarboxylation of pyruvate to acetyl CoA is also reduced in muscle-specific Bmal1-KO mice, indicating depleted glucose oxidation. These findings suggest that the muscle clock functions using glucose as the predominant energy substrate to fuel skeletal muscle at the light-to-dark transition. The findings of the DNA microarray analyses by Hodge et al indicated that genes involved in carbohydrate catabolism and storage peak early during the dark and the mid-active periods, respectively. ${ }^{59}$ This type of time-dependent gene expression is disrupted in musclespecific Bmall KO mice. These findings suggest that the intrinsic muscle clock temporally regulates genes involved in substrate utilization and storage and that a disrupted muscle clock diminishes the ability of skeletal muscle to maintain metabolic homeostasis.

\section{Application of circadian rhythms to maintenance of skeletal muscle function}

Pharmaceutical (e.g., testosterone, estrogen, and growth hormone) and non-pharmaceutical (e.g., resistance training, increased protein intake, and amino acid supplementation) approaches are applied to counteract muscle defects such as sarcopenia. ${ }^{60,61}$ Although pharmaceutical therapies can combat sarcopenia to some degree, resistance training when combined with amino acid supplementation is the most effective means of preventing and treating sarcopenia. ${ }^{62}$ However, the temporal specificity of these therapeutic methods for muscle defects has not been confirmed. In this section, we explain the studies on chronopharmacology, chrononutrition, and chronoexercise and discuss the potential of each for skeletal muscle health.

\section{Chronopharmacology}

Chronopharmacology aims to treat illnesses according to the endogenous biological rhythms that modify xenobiotic metabolism and cellular drug responses. ${ }^{63}$ Early studies of chronopharmacology published between the 1960s and 1970s identified several-fold changes in toxicity as a function of the time when a fixed dose of medicine was delivered. This is particularly important for treating cancer and inflammation. ${ }^{63}$ The recent development of programmable drug-delivery pumps has enabled anti-cancer drug release in specific tissues at selective time points with minimal or no nursing intervention. ${ }^{64,65} \mathrm{New}$ drug formulations have also been developed to control the timing of drug release in the body. For example, capsules of montelukast sodium, a drug to treat asthma, are administered before bedtime, and its formulation enables pulsatile release to prevent episodic asthma attacks in the early morning. ${ }^{66}$ Modifications in dosing time have 
also progressed. One type of conventional chronotherapy for hypertension aimed to attenuate the morning increase of blood pressure and thus medications such as angiotensin II inhibitors and calcium channel blockers were taken in the morning. However, a number of clinical trials failed to substantiate the merit of targeting the morning to decrease risk of cardiovascular diseases. ${ }^{67}$ Recent clinical trials showed that the ingestion of these agents at bedtime could shift $24 \mathrm{~h}$ blood pressure profiles toward normal and reduce the risk of vascular disease incidents. ${ }^{68-71}$

To our knowledge, pharmacological interventions for sarcopenia and muscle defects have not been proven. As an example of chronopharmacology to treat skeletal muscle mass loss, we showed that muscle atrophy as a side effect of glucocorticoid treatment can be attenuated by optimizing the dosing schedule. ${ }^{72}$ Muscle atrophy was more extreme in mice injected intraperitoneally with $10 \mathrm{mg} / \mathrm{kg}$ dexamethasone (a synthetic glucocorticoid), at ZT1 (inactive period) than at ZT13 (active period), when endogenous glucocorticoid release is lowest and highest during the day, respectively. ${ }^{72}$ Dexamethasone similarly decreased the weight of the spleen at both dosing times, indicating that the immunosuppressive effect of dexamethasone is time independent. ${ }^{72}$ These findings suggested that circadian rhythms should be considered during various types of therapy to retain skeletal muscle mass. We postulate that the molecular circadian clock was involved in these findings because the attenuation of muscle atrophy induced by time-dependent dosing with dexamethasone was abolished in $\mathrm{Clk} / \mathrm{Clk}$ mutant mice. ${ }^{72}$ However, we could not determine whether the dosing time-dependent attenuation of muscle atrophy was regulated directly by the muscle clock, or indirectly by molecular clocks in other organs, because Clock gene was mutated throughout the body in $C l k / C l k$ mutant mice. Further study using muscle specific-Bmall KO mice is needed to establish the role of muscle molecular clock on dosing time-dependent sensitivity to drugs.

\section{Chrononutrition}

Evidence of interaction between the circadian clock machinery and food intake/exercise supports the notion that chrononutrition and chronoexercise have therapeutic value. ${ }^{73,74}$ Chrononutrition encompasses the timing of food intake and the contribution of food components to the maintenance of health, and the timing of food intake or contribution of food components to rapid changes in or the resetting of circadian clock systems. ${ }^{74,75}$ Factors such as caffeine, nobiletin, and resveratrol in foods can change molecular and/or behavioral circadian rhythms. ${ }^{76}$ The relationship between the timing of food intake and skeletal muscle health remains controversial. Several studies have shown that the timing of dietary protein intake contributes to the acceleration of muscle protein synthesis in humans. Mamerow et al used a 7-day crossover feeding test to examine the effect of protein distribution among three meals per day in healthy adult men and women (age, 36.9 \pm 3.1 years; body mass index [BMI], $25.7 \pm 0.8) .{ }^{77}$ The participants consumed isoenergetic and isonitrogenous diets containing evenly or unevenly distributed amounts of protein at breakfast, lunch, and dinner $(31.5 \pm 1.3,29.9 \pm 1.6$, and $32.7 \pm 1.6 \mathrm{~g}$ vs. $10.7 \pm 0.8,16.0 \pm 0.5$, and $63.4 \pm 3.7 \mathrm{~g}$ protein). The rate of muscle protein synthesis on day 7 was $25 \%$ higher in the group that consumed evenly distributed protein. ${ }^{77}$ Murphy et al implemented a randomized trial that included overweight and obese older men (age, $66 \pm 4$ years; BMI, $31 \pm 5$ ). ${ }^{78}$ One group was given a diet with evenly distributed protein across all daily meals ( $\sim 25 \%$ of total protein intake at breakfast, lunch, dinner, and pre-bedtime snack). The other group received most of the dietary protein at dinnertime (about $7 \%$ of total protein intake at breakfast, $17 \%$ at lunch, $72 \%$ at dinner, and $4 \%$ as a pre-bedtime snack). One month later, myofibrillar protein synthesis was stimulated more by the diet with evenly than with unevenly distributed dietary protein in both the presence and absence of resistance training. ${ }^{78}$ Although neither study determined precise muscle weight and force, both showed that skewed dietary protein intake with the highest consumption of dietary protein in the evening stimulated less muscle-protein synthesis than a balanced distribution of daily protein intake. Whether or not the molecular clock in skeletal muscle is relevant to the different rates of muscle protein synthesis caused by the distribution of dietary protein intake remains unknown. The absorption and metabolism of dietary protein in other organs might cause time-dependent acceleration of protein synthesis in skeletal muscle, because the activity and expression of the intestinal $\mathrm{H}^{+}$peptide cotransporter PEPT1 is diurnally regulated. ${ }^{79,80}$ Lipton et al indicated that hepatic BMAL1 is rhythmically phosphorylated by the mTOR-effector kinase S6K1 and associated with translational machinery, suggesting that BMAL1 regulates the oscillation of protein synthesis independently of its role in transcriptional regulation. ${ }^{58}$ Further study is required to elucidate whether skeletal muscle protein synthesis oscillates in a circadian manner and whether it is relevant to muscle clock function. To understand the optimal timing for dietary protein intake to accelerate muscle protein synthesis would be useful to help patients with sarcopenia gain muscle mass. 


\section{Chronoexercise}

Chronoexercise is the study of the role of exercise timing in health maintenance and the effects of exercise on resetting the circadian clock system. Skeletal muscle torque, strength, and power in humans are higher during the late afternoon than during the morning, and this seems to be a result of muscle changes rather than central and systemic effects. ${ }^{81}$ As well as muscle performance is increased in the afternoon than in the morning, some groups have investigated whether training at a specific time of day could maximally increase muscle strength. ${ }^{81}$ Souissi et al examined the effect of regular training in the morning (between 07:00 and 08:00) or in the evening (between 17:00 and 18:00) for 6 weeks in male university students (age, $19 \pm 1.2$ years; height, $1.8 \pm 0.03 \mathrm{~m}$; body mass, $68.8 \pm 4.4 \mathrm{~kg}){ }^{82}$ The results showed that muscle power when measured in the morning was greater than that measured in the evening in the group that trained in the morning. On the other hand, muscle power when measured in the evening increased more in the group that trained in the evening, suggesting that adaptation to strength training is greater at the time of day when training routinely occurs ${ }^{82}$ Sedliak et al also evaluated the effect of training in the morning (between 07:00 and 09:00) or the evening (between 17:00 and 19:00) for 10 weeks on muscle strength and electromyographic (EMG) activity in healthy participants (morning group: age, $32 \pm 9$ years; height, $1.79 \pm 0.09 \mathrm{~m}$; body mass, $82.6 \pm 8.5 \mathrm{~kg}$; evening group: age, $33 \pm 7$ years; height, $1.80 \pm 0.07 \mathrm{~m}$; body mass, $79.9 \pm 11.3 \mathrm{~kg}$ ). ${ }^{83}$ Muscle strength increased when measured during the morning in the group that trained in the morning and increased when measured during the evening in the group that trained in the evening. However, EMG activity during the measurement of muscle strength did not reveal any time-of-day-specific adaption, suggesting that peripheral/muscle adaptation rather than neural adaptation is the main source of temporal specificity in strength training. ${ }^{83}$ Sedliak et al measured muscle volume to determine the effects of training-induced muscle hypertrophy at a specific time of day for 10 weeks in men. ${ }^{84}$ The results showed that 10 weeks of training in the morning or in the evening increased the cross-sectional area of the quadriceps femoris muscle by $2.7 \%$ and $3.5 \%$, respectively, but the difference was not significant. ${ }^{84}$ Adaptation to training is temporally specific to increase muscle strength, but not muscle volume, in adult humans ${ }^{83,84}$ Whether or not a specific time of training would improve sarcopenia in aged populations should be determined. The relevance of molecular clocks to a time-dependent effect of exercise also remains unknown. Adaptation to chronic exercise in mice does not require CLOCK protein because voluntary wheel running for
8 weeks improves the endurance capacity of $\mathrm{Clk} / \mathrm{Clk}$ mutant mice, although they had a lower mitochondrial content than wild-type mice. ${ }^{85}$ Sasaki et al indicated that the feeding phase was advanced and delayed by evening and morning exercise, respectively, in mice subjected to scheduled exercise for a period of $4 \mathrm{~h}$ during the early dark period or the late dark period using a running wheel..$^{45}$ The feeding pattern elicited by the scheduled exercise may modify skeletal muscle function. ${ }^{45}$ Further investigation is required to determine the role of molecular clocks in the effects of time-specific improvements in muscle performance caused by exercise.

\section{Conclusion}

Recent epidemiological studies have suggested that disruption of the circadian clock by environmental and biological factors such as aging, shift work, jet lag, and sleep disorders might contribute to the prevalence of diseases associated with skeletal muscle such as sarcopenia and metabolic disorders. ${ }^{2,3}$ A direct relationship between circadian rhythm disruption and skeletal muscle disease has not been identified in humans. However, several basic studies of different mouse models of clock gene deficiency have found that molecular clock disruption affects skeletal muscle physiology. The phenotype of muscle-specific Bmall KO mice uses less glucose as energy fuel, suggesting that the muscle clock functions using glucose as the predominant energy substrate at the light-todark transition in adult mice. ${ }^{50}$ On the other hand, clock gene disruption hardly affects muscle architecture and strength in young and adult mice. ${ }^{50,52,56}$ The effects of muscle clock disruption might be highlighted by aging, since the skeletal muscle of aged im-Bmal $\mathrm{KO}$ mice is fibrotic, has reduced force, and is accompanied by bone calcification, although such phenotypes are not evident in younger im-Bmal KO mice. ${ }^{52}$ Aging-related systemic changes such as the denervation of motor units, oxidative damage, as well as anabolic resistance and molecular clock disruption caused by shift work, jet lag, and sleep disorders might additively increase risk for poor skeletal muscle health among aged populations.

Treatment strategies for skeletal muscle-related diseases such as sarcopenia and metabolic diseases are mainly based on exercise and adequate protein intake. ${ }^{62}$ Consideration of the circadian clock machinery might help to render these approaches more effective for retaining skeletal muscle health because their effects might be time dependent, although this has not yet been directly proven. Several studies have found that exercise and several nutrients can alter the circadian phase and amplitude of clock gene expression, ${ }^{74,76,86}$ which implies that intervention could affect the circadian rhythms 
of skeletal muscle. The best timing for exercise and nutrition intake should differ among individuals. These facts should be taken into consideration when creating therapeutic strategies for sarcopenia and metabolic diseases, and maintaining a finely tuned molecular clock rhythm might be one factor involved in correcting these conditions.

\section{Acknowledgments}

This study was supported by operational subsidies from AIST; R Nakao (JP15K16499) was funded by JSPS KAKENHI and K Oishi (JP16K00940) was funded by the Ministry of Education, Culture, Sports, Science, and Technology (MEXT) of Japan.

\section{Disclosure}

The authors report no conflicts of interest in this work.

\section{References}

1. Bowen TS, Schuler G, Adams V. Skeletal muscle wasting in cachexia and sarcopenia: molecular pathophysiology and impact of exercise training. J Cachexia Sarcopenia Muscle. 2015;6(3):197-207.

2. Yu JH, Yun CH, Ahn JH, et al. Evening chronotype is associated with metabolic disorders and body composition in middle-aged adults. $J$ Clin Endocrinol Metab. 2015;100(4):1494-1502.

3. Di Lorenzo L, De Pergola G, Zocchetti C, et al. Effect of shift work on body mass index: results of a study performed in 319 glucose-tolerant men working in a Southern Italian industry. Int J Obes Relat Metab Disord. 2003;27(11):1353-1358.

4. Stephan FK, Zucker I. Circadian rhythms in drinking behavior and locomotor activity of rats are eliminated by hypothalamic lesions. Proc Natl Acad Sci U S A. 1972;69(6):1583-1586.

5. Drucker-Colin R, Aguilar-Roblero R, Garcia-Hernandez F, FernandezCancino F, Bermudez Rattoni F. Fetal suprachiasmatic nucleus transplants: diurnal rhythm recovery of lesioned rats. Brain Res. 1984;311(2): 353-357.

6. Ralph MR, Foster RG, Davis FC, Menaker M. Transplanted suprachiasmatic nucleus determines circadian period. Science. 1990;247(4945):975-978.

7. Colwell CS, Foster RG. Photic regulation of Fos-like immunoreactivity in the suprachiasmatic nucleus of the mouse. J Comp Neurol. 1992;324(2):135-142.

8. Meijer JH, Schwartz WJ. In search of the pathways for light-induced pacemaker resetting in the suprachiasmatic nucleus. $J$ Biol Rhythms. 2003;18(3):235-249.

9. Buhr ED, Takahashi JS. Molecular components of the mammalian circadian clock. Handb Exp Pharmacol. 2013(217):3-27.

10. Dardente H, Cermakian N. Molecular circadian rhythms in central and peripheral clocks in mammals. Chronobiol Int. 2007;24(2):195-213.

11. Eide EJ, Vielhaber EL, Hinz WA, Virshup DM. The circadian regulatory proteins BMAL1 and cryptochromes are substrates of casein kinase Iepsilon. J Biol Chem. 2002;277(19):17248-17254.

12. Lee C, Etchegaray JP, Cagampang FR, Loudon AS, Reppert SM. Posttranslational mechanisms regulate the mammalian circadian clock. Cell. 2001;107(7):855-867.

13. Lowrey PL, Shimomura K, Antoch MP, et al. Positional syntenic cloning and functional characterization of the mammalian circadian mutation tau. Science. 2000;288(5465):483-492.

14. Eide EJ, Woolf MF, Kang H, et al. Control of mammalian circadian rhythm by CKIepsilon-regulated proteasome-mediated PER2 degradation. Mol Cell Biol. 2005;25(7):2795-2807.
15. Busino L, Bassermann F, Maiolica A, et al. SCFFbxl3 controls the oscillation of the circadian clock by directing the degradation of cryptochrome proteins. Science. 2007;316(5826):900-904.

16. Preitner N, Damiola F, Lopez-Molina L, et al. The orphan nuclear receptor REV-ERBalpha controls circadian transcription within the positive limb of the mammalian circadian oscillator. Cell. 2002;110(2):251-260.

17. Sato TK, Panda S, Miraglia LJ, et al. A functional genomics strategy reveals Rora as a component of the mammalian circadian clock. Neuron. 2004;43(4):527-537.

18. Guillaumond F, Dardente H, Giguere V, Cermakian N. Differential control of Bmall circadian transcription by REV-ERB and ROR nuclear receptors. J Biol Rhythms. 2005;20(5):391-403.

19. Crumbley C, Burris TP. Direct regulation of CLOCK expression by REV-ERB. PLoS One. 2011;6(3):e17290.

20. Yamazaki $S$, Numano $R, A b e ~ M$, et al. Resetting central and peripheral circadian oscillators in transgenic rats. Science. 2000;288(5466):682-685.

21. Nagoshi E, Saini C, Bauer C, Laroche T, Naef F, Schibler U. Circadian gene expression in individual fibroblasts: cell-autonomous and self-sustained oscillators pass time to daughter cells. Cell. 2004;119(5):693-705.

22. Yoo SH, Yamazaki S, Lowrey PL, et al. PERIOD2::LUCIFERASE real-time reporting of circadian dynamics reveals persistent circadian oscillations in mouse peripheral tissues. Proc Natl Acad Sci U SA. 2004; 101(15):5339-5346.

23. Panda S, Antoch MP, Miller BH, et al. Coordinated transcription of key pathways in the mouse by the circadian clock. Cell. 2002;109(3):307-320.

24. Ueda HR, Chen W, Adachi A, et al. A transcription factor response element for gene expression during circadian night. Nature. 2002;418(6897):534-539.

25. Storch KF, Lipan O, Leykin I, et al. Extensive and divergent circadian gene expression in liver and heart. Nature. 2002;417(6884):78-83.

26. Miller BH, McDearmon EL, Panda S, et al. Circadian and CLOCKcontrolled regulation of the mouse transcriptome and cell proliferation. Proc Natl Acad Sci U S A. 2007;104(9):3342-3347.

27. Zylka MJ, Shearman LP, Weaver DR, Reppert SM. Three period homologs in mammals: differential light responses in the suprachiasmatic circadian clock and oscillating transcripts outside of brain. Neuron. 1998;20(6):1103-1110.

28. McCarthy JJ, Andrews JL, McDearmon EL, et al. Identification of the circadian transcriptome in adult mouse skeletal muscle. Physiol Genomics. 2007;31(1):86-95.

29. McWatters H, Dunlap JC, Millar AJ. Circadian biology: clocks for the real world. Curr Biol. 1999;9(17):R633-R635.

30. Roenneberg T, Merrow M. Circadian clocks - from genes to complex behaviour. Reprod Nutr Dev. 1999;39(3):277-294.

31. Damiola F, Le Minh N, Preitner N, Kornmann B, Fleury-Olela F, Schibler U. Restricted feeding uncouples circadian oscillators in peripheral tissues from the central pacemaker in the suprachiasmatic nucleus. Genes Dev. 2000;14(23):2950-2961.

32. Stokkan KA, Yamazaki S, Tei H, Sakaki Y, Menaker M. Entrainment of the circadian clock in the liver by feeding. Science. 2001;291(5503):490-493.

33. Guo H, Brewer JM, Champhekar A, Harris RB, Bittman EL. Differential control of peripheral circadian rhythms by suprachiasmatic-dependent neural signals. Proc Natl Acad Sci U S A. 2005;102(8):3111-3116.

34. Hara R, Wan K, Wakamatsu H, et al. Restricted feeding entrains liver clock without participation of the suprachiasmatic nucleus. Genes Cells. 2001;6(3):269-278.

35. Yasumoto Y, Hashimoto C, Nakao R, et al. Short-term feeding at the wrong time is sufficient to desynchronize peripheral clocks and induce obesity with hyperphagia, physical inactivity and metabolic disorders in mice. Metabolism. 2016;65(5):714-727.

36. Oishi K, Yasumoto Y, Higo-Yamamoto S, Yamamoto S, Ohkura N. Feeding cycle-dependent circulating insulin fluctuation is not a dominant Zeitgeber for mouse peripheral clocks except in the liver: differences between endogenous and exogenous insulin effects. Biochem Biophys Res Commun. 2017;483(1):165-170. 
37. Reebs SG, Mrosovsky N. Effects of induced wheel running on the circadian activity rhythms of Syrian hamsters: entrainment and phase response curve. J Biol Rhythms. 1989;4(1):39-48.

38. Marchant EG, Mistlberger RE. Entrainment and phase shifting of circadian rhythms in mice by forced treadmill running. Physiol Behav. 1996;60(2):657-663.

39. Zambon AC, McDearmon EL, Salomonis N, et al. Time- and exercisedependent gene regulation in human skeletal muscle. Genome Biol. 2003;4(10):R61

40. Wolff G, Esser KA. Scheduled exercise phase shifts the circadian clock in skeletal muscle. Med Sci Sports Exerc. 2012;44(9):1663-1670.

41. Hawley JA, Hargreaves M, Joyner MJ, Zierath JR. Integrative biology of exercise. Cell. 2014;159(4):738-749.

42. Balsalobre A, Brown SA, Marcacci L, et al. Resetting of circadian time in peripheral tissues by glucocorticoid signaling. Science. 2000; 289(5488):2344-2347.

43. Yasumoto Y, Nakao R, Oishi K. Free access to a running-wheel advances the phase of behavioral and physiological circadian rhythms and peripheral molecular clocks in mice. PLoS One. 2015;10(1):e0116476.

44. Droste SK, Gesing A, Ulbricht S, Muller MB, Linthorst AC, Reul JM. Effects of long-term voluntary exercise on the mouse hypothalamicpituitary-adrenocortical axis. Endocrinology. 2003;144(7):3012-3023.

45. Sasaki H, Hattori Y, Ikeda Y, et al. Phase shifts in circadian peripheral clocks caused by exercise are dependent on the feeding schedule in PER2::LUC mice. Chronobiol Int. 2016;33(7):849-862.

46. Nakao R, Yamamoto S, Horikawa K, et al. Atypical expression of circadian clock genes in denervated mouse skeletal muscle. Chronobiol Int. 2015;32(4):486-496.

47. Dyar KA, Ciciliot S, Tagliazucchi GM, et al. The calcineurin-NFAT pathway controls activity-dependent circadian gene expression in slow skeletal muscle. Mol Metab. 2015;4(11):823-833.

48. Kondratov RV, Kondratova AA, Gorbacheva VY, Vykhovanets OV, Antoch MP. Early aging and age-related pathologies in mice deficient in BMAL1, the core componentof the circadian clock. Genes Dev. 2006;20(14):1868-1873.

49. Andrews JL, Zhang X, McCarthy JJ, et al. CLOCK and BMAL1 regulate MyoD and are necessary for maintenance of skeletal muscle phenotype and function. Proc Natl Acad Sci US A. 2010;107(44):19090-19095.

50. Dyar KA, Ciciliot S, Wright LE, et al. Muscle insulin sensitivity and glucose metabolism are controlled by the intrinsic muscle clock. Mol Metab. 2014;3(1):29-41.

51. Nakao R, Shimba S, Oishi K. Muscle Bmal1 is dispensable for the progress of neurogenic muscle atrophy in mice. J Circadian Rhythms. 2016;14(1):6.

52. Schroder EA, Harfmann BD, Zhang X, et al. Intrinsic muscle clock is necessary for musculoskeletal health. J Physiol. 2015;593(24):5387-5404.

53. Nyberg M, Hellsten Y. Reduced blood flow to contracting skeletal muscle in ageing humans: is it all an effect of sand through the hourglass? J Physiol. 2016;594(8):2297-2305.

54. Gomes MJ, Martinez PF, Pagan LU, et al. Skeletal muscle aging: influence of oxidative stress and physical exercise. Oncotarget. 2017;8(12):20428-20440.

55. Jacobi D, Liu S, Burkewitz K, et al. Hepatic Bmal1 regulates rhythmic mitochondrial dynamics and promotes metabolic fitness. Cell Metab. 2015;22(4):709-720.

56. Yang G, Chen L, Grant GR, et al. Timing of expression of the core clock gene Bmall influences its effects on aging and survival. Sci Transl Med. 2016;8(324):324ra316.

57. Dolatshad H, Cary AJ, Davis FC. Differential expression of the circadian clock in maternal and embryonic tissues of mice. PLoS One. 2010;5(3):e9855.

58. Lipton JO, Yuan ED, Boyle LM, et al. The circadian protein BMAL1 regulates translation in response to $\mathrm{S} 6 \mathrm{~K} 1$-mediated phosphorylation. Cell. 2015;161(5):1138-1151.

59. Hodge BA, Wen Y, Riley LA, et al. The endogenous molecular clock orchestrates the temporal separation of substrate metabolism in skeletal muscle. Skelet Muscle. 2015;5:17.
60. Doherty TJ. Invited review: aging and sarcopenia. J Appl Physiol. 2003;95(4):1717-1727.

61. Lang T, Streeper T, Cawthon P, Baldwin K, Taaffe DR, Harris TB. Sarcopenia: etiology, clinical consequences, intervention, and assessment. Osteoporos Int. 2010;21(4):543-559.

62. Wakabayashi H, Sakuma K. Comprehensive approach to sarcopenia treatment. Curr Clin Pharmacol. 2014;9(2):171-180.

63. Ballesta A, Innominato PF, Dallmann R, Rand DA, Levi FA. Systems chronotherapeutics. Pharmacol Rev. 2017;69(2):161-199.

64. Levi F, Okyar A, Dulong S, Innominato PF, Clairambault J. Circadian timing in cancer treatments. Annu Rev Pharmacol Toxicol. 2010;50:377-421.

65. Levi FA, Boige V, Hebbar M, et al. Conversion to resection of liver metastases from colorectal cancer with hepatic artery infusion of combined chemotherapy and systemic cetuximab in multicenter trial OPTILIV. Ann Oncol. 2016;27(2):267-274.

66. Ranjan OP, Nayak UY, Reddy MS, Dengale SJ, Musmade PB, Udupa $\mathrm{N}$. Osmotically controlled pulsatile release capsule of montelukast sodium for chronotherapy: statistical optimization, in vitro and in vivo evaluation. Drug Deliv. 2014;21(7):509-518.

67. Smolensky MH, Hermida RC, Ayala DE, Portaluppi F. Bedtime hypertension chronotherapy: concepts and patient outcomes. Curr Pharm Des. 2015;21(6):773-790.

68. Hermida RC, Ayala DE, Mojon A, Fernandez JR. Influence of circadian time of hypertension treatment on cardiovascular risk: results of the MAPEC study. Chronobiol Int. 2010;27(8):1629-1651.

69. Hermida RC, Ayala DE, Fernandez JR, Portaluppi F, Fabbian F, Smolensky MH. Circadian rhythms in blood pressure regulation and optimization of hypertension treatment with ACE inhibitor and ARB medications. Am J Hypertens. 2011;24(4):383-391.

70. Hermida RC, Ayala DE, Mojon A, Fernandez JR. Bedtime dosing of antihypertensive medications reduces cardiovascular risk in CKD. J Am Soc Nephrol. 2011;22(12):2313-2321.

71. Roush GC, Fapohunda J, Kostis JB. Evening dosing of antihypertensive therapy to reduce cardiovascular events: a third type of evidence based on a systematic review and meta-analysis of randomized trials. J Clin Hypertens. 2014;16(8):561-568.

72. Nakao R, Yamamoto S, Yasumoto Y, Oishi K. Dosing scheduledependent attenuation of dexamethasone-induced muscle atrophy in mice. Chronobiol Int. 2014;31(4):506-514.

73. Oike H, Oishi K, Kobori M. Nutrients, clock genes, and chrononutrition. Current Nutr Rep. 2014;3:204-212.

74. Tahara Y, Shibata S. Chronobiology and nutrition. Neuroscience. 2013;253:78-88.

75. Johnston JD, Ordovas JM, Scheer FA, Turek FW. Circadian rhythms, metabolism, and chrononutrition in rodents and humans. Adv Nutr. 2016;7(2):399-406.

76. Oike H. Modulation of circadian clocks by nutrients and food factors. Biosci Biotechnol Biochem. 2017;81(5):863-870.

77. Mamerow MM, Mettler JA, English KL, et al. Dietary protein distribution positively influences $24-\mathrm{h}$ muscle protein synthesis in healthy adults. $J$ Nutr. 2014;144(6):876-880.

78. Murphy CH, Churchward-Venne TA, Mitchell CJ, et al. Hypoenergetic diet-induced reductions in myofibrillar protein synthesis are restored with resistance training and balanced daily protein ingestion in older men. Am J Physiol Endocrinol Metab. 2015;308(9):E734-E743.

79. Pan X, Terada T, Irie M, Saito H, Inui K. Diurnal rhythm of H+-peptide cotransporter in rat small intestine. Am J Physiol Gastrointest Liver Physiol. 2002;283(1):G57-G64.

80. Pan X, Terada T, Okuda M, Inui K. Altered diurnal rhythm of intestinal peptide transporter by fasting and its effects on the pharmacokinetics of ceftibuten. J Pharmacol Exp Ther. 2003;307(2):626-632.

81. Zhang X, Dube TJ, Esser KA. Working around the clock: circadian rhythms and skeletal muscle. J Appl Physiol. 2009;107(5):1647-1654.

82. Souissi N, Gauthier A, Sesboue B, Larue J, Davenne D. Effects of regular training at the same time of day on diurnal fluctuations in muscular performance. J Sports Sci. 2002;20(11):929-937. 
83. Sedliak M, Finni T, Peltonen J, Hakkinen K. Effect of time-of-dayspecific strength training on maximum strength and EMG activity of the leg extensors in men. $J$ Sports Sci. 2008;26(10):1005-1014.

84. Sedliak M, Finni T, Cheng S, Lind M, Hakkinen K. Effect of timeof-day-specific strength training on muscular hypertrophy in men J Strength Cond Res. 2009;23(9):2451-2457.
85. Pastore S, Hood DA. Endurance training ameliorates the metabolic and performance characteristics of circadian Clock mutant mice. $J$ Appl Physiol. 2013;114(8):1076-1084.

86. Aoyama S, Shibata S. The role of circadian rhythms in muscular and osseous physiology and their regulation by nutrition and exercise. Front Neurosci. 2017;11:63.

\section{Publish your work in this journal}

ChronoPhysiology and Therapy is an international, peer-reviewed open access journal focusing on research into the cyclic variations and rhythmicity in physiological processes in the body and the research and development and optimal timing of administration of therapeutic targets to achieve improved outcomes and quality of life for the patient. The manuscript management system is completely online and includes a very quick and fair peer-review system. Visit http://www.dovepress.com/ testimonials.php to read real quotes from published authors. 